\title{
The expression and correlation between chemokine CCL7 and ABCE1 in non-small cell lung cancer
}

\author{
ZHUO WU ${ }^{1}$, YE TIAN ${ }^{1}$, QIAN YU ${ }^{1}$, HONGYUAN LI ${ }^{2}$, ZHIQIANG TIAN ${ }^{1}$, \\ HONGFANG JIANG $^{3}$, DALI TIAN ${ }^{1}$ and XUEYING YANG ${ }^{1}$ \\ Departments of ${ }^{1}$ Thoracic Surgery and ${ }^{2}$ Oncology, The Fourth Affiliated Hospital of \\ China Medical University, Shenyang, Liaoning 110032; ${ }^{3}$ Department of Geriatrics, \\ Shengjing Hospital of China Medical University, Shenyang, Liaoning 110001, P.R. China
}

Received February 10, 2018; Accepted June 22, 2018

DOI: $10.3892 /$ etm.2018.6568

\begin{abstract}
Lung cancer is a malignant disease, and has the highest incidence and mortality worldwide. Lung cancer is also a popular subject in the field of cancer research. The molecular mechanisms of lung cancer development, invasion and metastasis need to be determined to prolong survival times and improve the quality of life. Recent studies have demonstrated that ATP-binding cassette sub-family E member 1 (ABCE1) is one of the factors that contributes to the development and metastasis of lung cancer, but the specific mechanism of this phenomenon remains unclear. A polymerase chain reaction microarray was used in the present study to screen for chemokine (C-C motif) ligand 7 (CCL7) expression in cell lines that highly expressed ABCE1, and the results showed that CCL7 was highly expressed in $\mathrm{H} 1299$ cells $(\mathrm{P}<0.01)$. The expression of CCL7 and ABCE1 in lung cancer tissues obtained from 30 patients with non-small cell lung cancer (NSCLC) was higher than that in adjacent normal lung tissues $(\mathrm{P}<0.01)$, and a positive correlation between the expression levels of the two genes in NSCLC was observed. These findings indicate that ABCE1 is involved in the development and progression of lung cancer through the CCL7 signaling pathway.
\end{abstract}

\section{Introduction}

ATP-binding cassette sub-family E member 1 (ABCE1) is a member of the ATP-binding cassette superfamily (1). ABCE1 acts as an RNase L inhibitor or host protein (HP) 68 and has been reported to participate in HIV-1 capsid assembly (2).

Correspondence to: Professor Dali Tian or Professor Xueying Yang, Department of Thoracic Surgery, The Fourth Affiliated Hospital of China Medical University, 4 Chongshan East Road, Shenyang, Liaoning 110032, P.R. China

E-mail: dalitian2013@yeah.net

E-mail: yangxy1974@yeah.net

Key words: ABCE1, CCL7, non-small cell lung cancer, invasion, metastasis
After the ABCE1 gene was silenced in the human small cell lung cancer cell line NCI-H446 using RNAi technology, in vitro cell biology experiments, including cell adhesion, wound healing, migration, and invasion experiments, were performed. These assays demonstrated that the migration and invasiveness of small cell lung cancer cells were significantly inhibited (3). ABCE1 was confirmed to be one of the key factors that promotes the development and metastasis of lung cancer following the inoculation of nude mice with the lung adenocarcinoma (AC) cell line LTEP-a-2, which has upregulated ABCE1 expression (4).

Chemokine (C-C motif) ligand 7, which was also known as monocyte chemotactic factor- 3 for a long period of time, can induce the majority of immune inflammatory cells, especially monocytes (5). CCL7 plays an important role in various pathologies, including cancers, auto-immune diseases and chronic inflammation (6). Monocytes have strong chemotactic ability towards tumor-associated macrophages (TAMs), and several chemokines, including CCL7, interact with cancer-associated fibroblasts (CAFs), which can influence the tumor microenvironment and promote tumor angiogenesis and infiltration by TAMs (7).

The relationship between ABCE1 and chemokine CCL7 in lung cancer has never been reported. This study attempted to determine the relationship between $\mathrm{ABCE} 1$ and chemokine CCL7 in lung cancer using a PCR microarray and immunohistochemistry to provide a new basis for the roles of ABCE1 and CCL7 in the pathogenesis of lung cancer.

\section{Materials and methods}

Cell culture and lentiviral packaging vector transfection. The lung cancer cell line H1299 was purchased from the Shanghai Chinese Academy of Science. The H1299 cells were cultured in RPMI-1640 medium supplemented with $10 \%$ fetal bovine serum under the following conditions: $37^{\circ} \mathrm{C}, 5 \% \mathrm{CO}_{2}$, and an aseptic environment. The culture medium was changed every 1 or 2 days. After they reached confluence, the cells were digested with $0.25 \%$ trypsin for subculture, cryopreservation and lentivirus transfection.

The lentiviral packaging vector that overexpressed ABCE1 was purchased from JiKai Gene Chemical Technology 
(Shanghai, China). The elements sequence incorporated into the GV358 vector was Ubi-MCS-3FLAG-SV40-EGFP-IRESpuromycin. The restriction enzyme site was located in AgeI. Recombinant clones were screened by puromycin, and the constructs expressed green fluorescent protein (GFP) reporter genes. The experimental cells were divided into an overexpression group and an empty vector group and were seeded in six-well plates. After the cells reached $30 \%$ confluence, culture medium with enhanced transfection reagent and polybrene $(5 \mu \mathrm{g} / \mathrm{ml})$ were added to the wells for the transfection experiments; the amount of virus added was calculated based on the pre-experimental values of the multiplicity of infection (MOI). A fluorescence microscope was used to observe the transfection efficiency, which was $80 \%$ or greater.

$R T^{2}$ Profiler $^{T M}$ PCR Array. Total RNA in the cells was extracted using a TaKaRa RNA extraction kit (TaKaRa Bio Inc., Dalian, China) and was stored at $-80^{\circ} \mathrm{C}$. An RT ${ }^{2}$ First Strand Kit (Qiagen GmbH, Hilden, Germany) was used to synthesize cDNA, and a comparative study was performed using an $\mathrm{RT}^{2}$ Profiler ${ }^{\mathrm{TM}}$ PCR Array Human Tumor Metastasis (PAHS-028Z) chip and RT-SYBR-Green Master Mix. qPCR was performed using an ABI7500 PCR system (Applied Biosystems; Thermo Fisher Scientific, Inc., Waltham, MA, USA). A number of housekeeping genes served as internal controls for sample normalization, and the $2^{-\Delta \Delta C q}$ values were compared (8).

Western blotting. After the H1299 cells were transfected with the lentiviral vector, total protein was extracted. The BCA method was used to determine the protein concentration, and $30 \mu \mathrm{g}$ of total protein was loaded onto a $10 \%$ SDS-PAGE gel for protein electrophoresis and then transferred onto a PVDF film, which was incubated with an ABCE1 rabbit anti-human monoclonal antibody (1:2,000 dilution; Abcam, Cambridge, MA, USA), a CCL7 rabbit anti-human polyclonal antibody (1:1,000 dilution; Sigma-Aldrich; Merck KGaA, Darmstadt, Germany) and a GAPDH mouse anti-human polyclonal antibody (1:1,000 dilution; Abcam) overnight at $4^{\circ} \mathrm{C}$. After the membranes were incubated with the indicated secondary antibodies (goat anti-rabbit monoclonal antibody and goat anti-mouse monoclonal antibody, 1:1,000 dilution) for $2 \mathrm{~h}$ at room temperature, the bands were visualized with enhanced chemiluminescence (ECL kit; Thermo Fisher Scientific, Inc.) with dark room exposure and development. The gray values of the protein bands, which represented the relative expression levels of the proteins, were determined.

Patient selection and tissue specimens. Cancer tissues and adjacent normal tissues (NTs) (located more than $2 \mathrm{~cm}$ from the edge of the tumor) surgically resected from 30 patients with non-small cell lung cancer (NSCLC) in the Department of Thoracic Surgery, Fourth Affiliated Hospital of China Medical University (Shenyang, China), from 2014 to 2016 were embedded in paraffin. These patients, including 13 males and 17 females with an average age of 62.4 years (range, 52 to 78 years), did not receive preoperative radiotherapy; the group included 12 cases of squamous cell carcinoma (SCC) and 18 cases of AC.
Immunohistochemical analysis. The tissue specimens were sliced, baked for $2 \mathrm{~h}$, soaked in xylene for deparaffinization, subjected to benzene removal using $100 \%$ ethanol, and then subjected to gradient ethanol hydration before they were rinsed with $0.01 \mathrm{~mol} / \mathrm{l}$ phosphate-buffered saline (PBS). The slices were then incubated in $0.01 \mathrm{M}$ citric acid buffer $(\mathrm{pH}=6.0)$ for $20 \mathrm{~min}$ at $97^{\circ} \mathrm{C}$ for antigen retrieval. After endogenous peroxidase was blocked and the slides were incubated with non-immune animal serum at room temperature, each section was incubated with $50 \mu 1$ of the appropriate primary antibody (ABCE1 1:500; CCL7 1:250) in a humidified chamber overnight at $4^{\circ} \mathrm{C}$. After the sections were rinsed with PBS, a biotinylated secondary antibody was added to them, and the slides were incubated for $10 \mathrm{~min}$ at room temperature. Streptavidin peroxidase solution was then added to the slides, which were incubated for another $10 \mathrm{~min}$. Approximately $100 \mu \mathrm{l}$ of diaminobenzidine (DAB) liquid was added to each of the tissue sections, all of which were observed under a microscope for $10 \mathrm{~min}$ before the reaction was terminated. Hematoxylin solution was then added to the slides as a counterstain for $5 \mathrm{~min}$ to visualize the nuclei.

Cells with a brownish-yellow membrane and cytoplasm were considered positively stained. Ten continuous high-power fields (x400) in each slice were observed under a light microscope and given scores of $0,1,2$ or 3 points according to the color intensity; the average score was then recorded. Fields with a positive cell rate of $<5 \%, 5-25 \%, 26-50 \%, 51-75 \%$, or $>75 \%$ were given scores of $0,1,2,3$ or 4 points, respectively. Both scores were multiplied, and the final score was categorized as follows: A score of 0-2 points was considered negative (-), a score of 3-4 points was considered weakly positive $(+)$, a score of 5-8 points was considered moderately positive $(++)$, and a score of 9-12 points was considered strongly positive $(+++)$. In addition, $(++)$ and $(+++)$ were considered high expression, and $(-)$ and $(+)$ were considered low expression.

Quantitative PCR ( PCR). PCR was performed in a 96-well plate. Each well contained $20 \mu 1$ of the reaction system, which included $10 \mu \mathrm{l}$ of SYBR Premix Ex Taq II (Takara Biotechnology Co., Ltd., Dalian, China) and a total of $1.6 \mu \mathrm{l}$ of upstream and downstream primers. The primers were as follows: CCL7 primer: 5'-GACAAGAAAACCCAAACTCCA AAG-3' and 5'-TCAAAACCCACCAAAATCCA-3'; ABCE1 primer: 5'-CAGCCTTTGTTGTGGAACATGA-3' and 5'-ATT CGTGGCCTATAGTTGTTTGGA-3'; and GAPDH primer: 5'-CACAAGAAGGTGGTGAAGCAG-3' and 5'-AAAGGT GGAGGAGTGGGTGT-3'. PCR analysis was performed using an ABI7500 PCR reaction system.

Statistical analysis. The results of the qPCR Array: The $G A P D H$ gene was used as the internal control gene; the relative expression of the genes was calculated as $\Delta \mathrm{Cq}=\mathrm{Cq}_{\text {target }}$ gene- $\mathrm{Cq}_{\text {internal control }}$, and the difference between groups was calculated as $\Delta \Delta \mathrm{Cq}=\Delta \mathrm{Cq}_{\text {experimental group }}-\Delta \mathrm{Cq}_{\text {control group }}$. The relationship between the experimental and control groups was expressed as $2^{-\Delta \Delta \mathrm{Cq}}(8)$.

Statistical analysis software provided by Qiagen $\mathrm{GmbH}$ was used for statistical analysis and mapping. $\mathrm{P}<0.05$ was considered to indicate a statistically significant difference. 

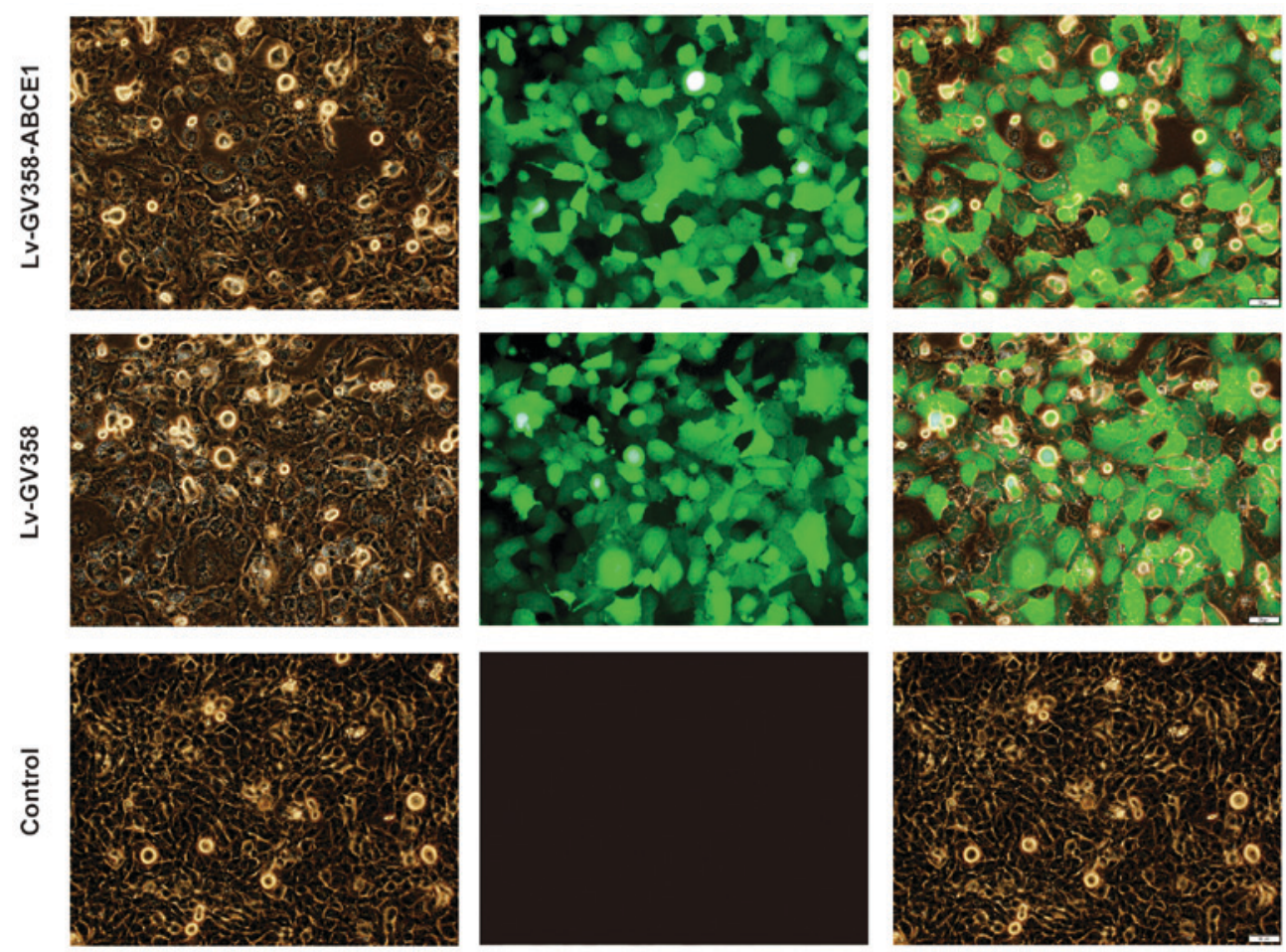

Figure 1. Fluorescence microscopic observation of $\mathrm{H} 1299$ cells $72 \mathrm{~h}$ after transfection with the ABCE1-overexpression lentiviral vector or empty vector (x400), The images in the first column are cells under bright field, the images in the second column are cells carrying green fluorescent protein under dark field, and the images in the third column are combined images from the first two columns. These images demonstrate that the transfection efficiency was greater than $80 \%$. The last lane is samples from control cells. ABCE1, ATP-binding cassette transporter E1.

Western blotting: Black bands on the PVDF film, which indicated positive results, were scanned by a gel imaging system for quantitative analysis based on the gray values. The protein band of GAPDH was used as the control. SPSS21.0 statistical analysis software (SPSS, Inc., Chicago, IL, USA) was used for the analysis. Measurement data are presented as the mean \pm standard deviation and were analyzed using the paired sample t-test and one-way ANOVA with Tukey's post hoc test. $\mathrm{P}<0.05$ was considered to indicate a statistically significant difference.

Immunohistochemistry and qPCR: SPSS 21.0 statistical analysis software (SPSS, Inc.) was employed. Measurement data are presented as mean \pm standard deviation and were analyzed using the paired sample t-test. $\mathrm{P}<0.05$ was considered to indicate a statistically significant difference, and correlations were evaluated using Pearson correlation analysis.

\section{Results}

Transfection of the NSCLC cell line H1299 using a lentiviral vector with $A B C E 1$ overexpression. The $\mathrm{H} 1299$ cells were transfected with Eni.S and polybrene. As the LV-GV358-ABCE1 and LV-GV358 constructs contained GFP, the transfected cells were observed to have visible green fluorescence in the cytoplasm when viewed under a fluorescence microscope. The transfection efficiency was $80 \%$ or greater. No fluorescence was observed in the untreated control cells (Fig. 1).

Screening of ABCE1-related genes by a PCR Array chip. Total mRNA in the LV-GV358-ABCE1- and LV-GV358-transfected H1299 cells was extracted and then reverse transcribed to obtain cDNA fragments. Using the cDNA as the template, we used an $\mathrm{RT}^{2}$ Profiler ${ }^{\mathrm{TM}}$ PCR Array Human Tumor Metastasis chip for qPCR. The experiments were performed in triplicate, and in all, nine tumor metastasis-related genes were screened by statistical analysis. The difference in CCL7 expression was the most significant (Fig. 2; Table I), and the CCL7 gene was selected as the primary research target from the group of ABCE1-related metastasis genes.

Western blotting. The expression of CCL7 in LV-GV358-ABCE1-transfected H1299 cells $(0.73 \pm 0.019)$ was significantly higher than that in LV-GV358-transfected H1299 cells $(0.32 \pm 0.019)$ and normal H1299 cells $(0.35 \pm 0.021), \mathrm{P}<0.01$, and the expression of ABCE1 inLV-GV358-ABCE1-transfected H1299 cells $(0.56 \pm 0.016)$ was significantly higher than that in LV-GV358-transfected H1299 cells $(0.37 \pm 0.016)$ and normal H1299 cells (0.34 \pm 0.003$), \mathrm{P}<0.01$ (Fig. 3).

Immunohistochemistry. The expression of the CCL7 and ABCE1 proteins was mainly localized in the cytoplasm, but CCL7 was also expressed in some fibroblasts and capillary endothelial cells. The expression level of CCL7 in lung cancer tissues $(8.6 \pm 0.58)$ was higher than that in adjacent NTs (2.6 \pm 0.35$), \mathrm{P}<0.01$, and the expression level of $\mathrm{ABCE} 1$ in lung cancer tissues $(9.13 \pm 0.6)$ was higher than that in adjacent NTs (2.13 \pm 0.29$), \mathrm{P}<0.01$ (Fig. 4). The rate of positive CCL7 expression in lung cancer tissues was $70 \%$, and the rate of positive $\mathrm{ABCE} 1$ expression in lung cancer tissues was $87 \%$.

$q P C R$. The relative expression levels ( $\triangle \mathrm{Cq}$ values) of CCL7 mRNA in NSCLC tissues and adjacent tissues were 10.66 \pm 0.41 
Table I. Genes differentially expressed between ABCE1 overexpression and empty vector groups.

\begin{tabular}{llrr}
\hline Gene & \multicolumn{1}{c}{ Description } & Fold change & P-value \\
\hline CCL7 & Chemokine (C-C motif) ligand 7 & 12.49 & 0.0048 \\
TIMP3 & TIMP metallopeptidase inhibitor 3 & 5.63 & 0.0154 \\
CXCR2 & Chemokine (C-X-C motif) receptor 2 & 3.37 & 0.0338 \\
ETV4 & Ets variant 4 & 3.16 & 0.0401 \\
TNFSF10 & TNF superfamily member 10 & 3.11 & 0.0262 \\
SERPINE1 & Serpin peptidase inhibitor, clade E, member 1 & -5.41 & 0.0124 \\
CXCL12 & Chemokine (C-X-C motif) ligand 12 & -5.02 & 0.0228 \\
MMP11 & Matrix metallopeptidase 11 & -4.87 & 0.0250 \\
ITGA7 & Integrin, $\alpha 7$ & -4.76 & 0.0498
\end{tabular}

ABCE1, ATP-binding cassette sub-family E member 1.

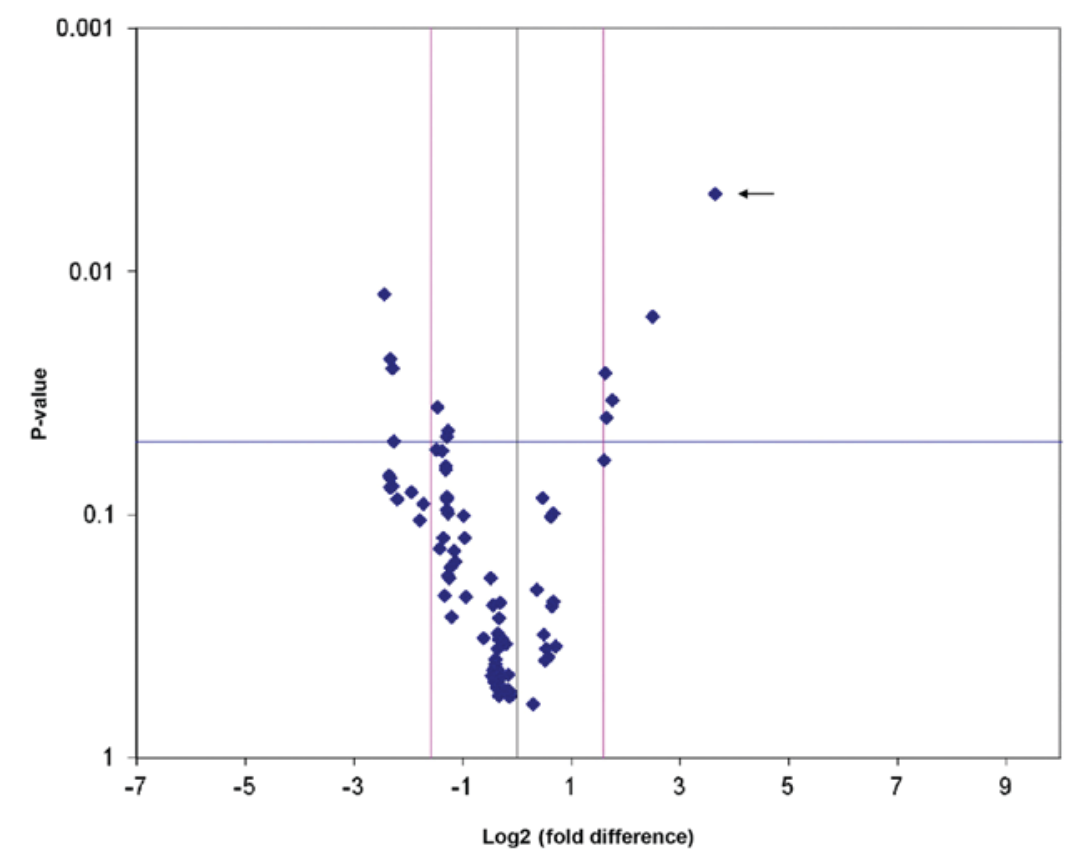

Figure 2. Volcano plot of gene expression differences, with the difference between the experimental data for the two groups as the $\mathrm{x}$-axis and the P-value as the $y$-axis. The blue line represents $\mathrm{P}<0.05$, and the arrow indicates CCL7. CCL7, chemokine (C-C motif) ligand 7.

and $13.93 \pm 0.39$, respectively, $\mathrm{P}<0.01$, and the $\Delta \mathrm{Cq}$ values of ABCE1 mRNA in NSCLC tissues and adjacent tissues were $8.29 \pm 0.33$ and $10.31 \pm 0.27$, respectively, $\mathrm{P}<0.01$. The mRNA expression of CCL7 was positively correlated with that of ABCE1 in NSCLC, with a Pearson correlation coefficient of $\mathrm{r}=0.6944, \mathrm{P}<0.01$ (Fig. 5).

\section{Discussion}

Due to its malignancy and threat to human health, lung cancer is a hot topic in the field of cancer research. The incidence and mortality of lung cancer are currently increasing annually, the age at onset is decreasing, and the disease is widespread and occurs worldwide (8). In recent years, with the development of molecular biology, more cancer genes and pathogenic mechanisms have been identified; however, the survival and disease remission rates of lung cancer are still low. Lymph node and organ metastases are important factors in determining the degree of malignancy in lung cancer; thus, further investigations into the mechanism of lung cancer metastasis are essential.

As a specific inhibitor of RNase and a key enzyme in the interferon-dependent 2-5A/RNase L pathway, ABCE1 plays an important physiological role in the regulation of the stability of cell RNA (9). ABCE1 also plays an important role in the initiation, extension and termination of eukaryotic protein translation, as well as in ribosome recycling $(10,11)$. Ren et al (12) found that ABCE1 was highly expressed in human lung $\mathrm{AC}$ and metastatic lymph nodes and was associated with clinical stage. Gao et al (13) observed that the expression levels of ABCE1 was correlated with histopathological type, but not with age, gender, the grade of tumor differentiation. In $\mathrm{AC}$, the expression level of $\mathrm{ABCE} 1$ protein were higher than that in the squamous carcinoma. Recently, a series of studies revealed that $\mathrm{ABCE} 1$ may be a new interaction protein for 
A

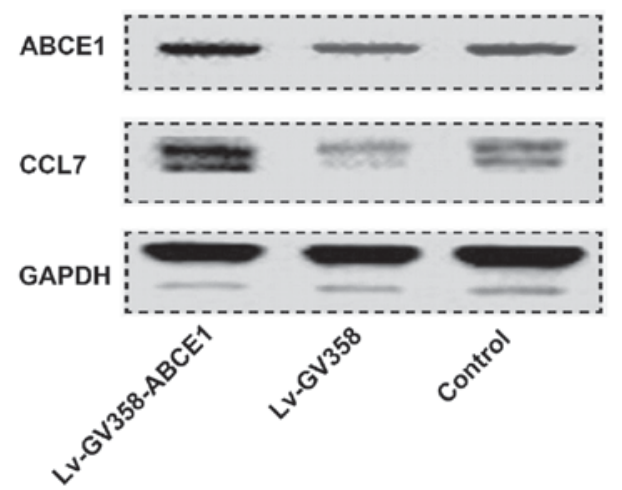

B

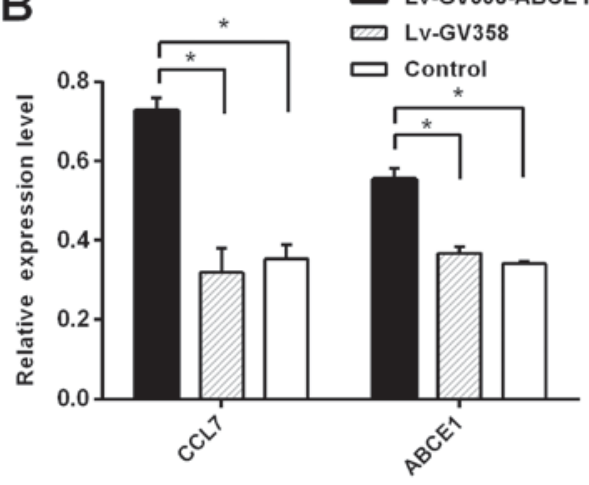

Figure 3. The western blotting results. (A) CCL7 and ABCE1 protein content in H1299 cells transfected with the ABCE1-overexpressing lentivirus was significantly higher than that in the empty vector control and normal control groups. (B) Histogram showing that the expression of CCL7 and ABCE1 in the ABCE1-upregulation group was higher than that in the empty vector and normal control groups, ${ }^{*} \mathrm{P}<0.01$. ABCE1, ATP-binding cassette sub-family $\mathrm{E}$ member 1; CCL7, chemokine (C-C motif) ligand 7.

A

\section{$\mathrm{AC}$}

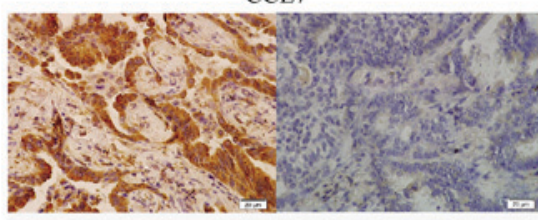

$\mathrm{CC}$

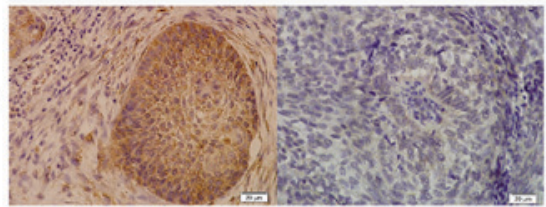

NT

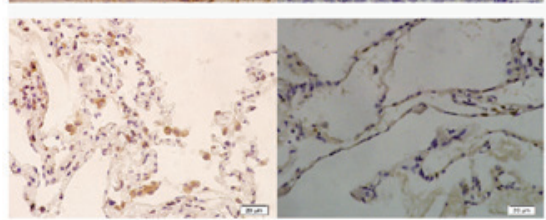

B

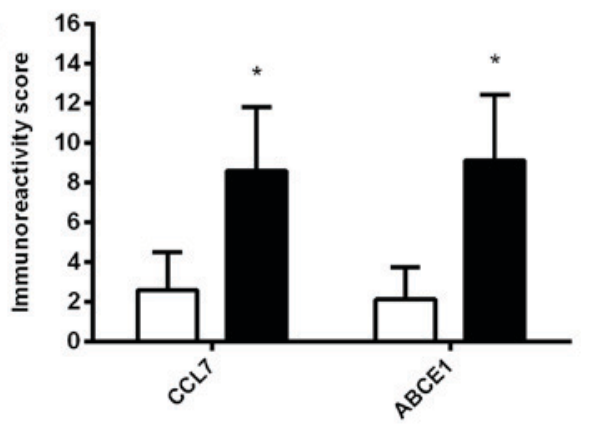

ABCE 1
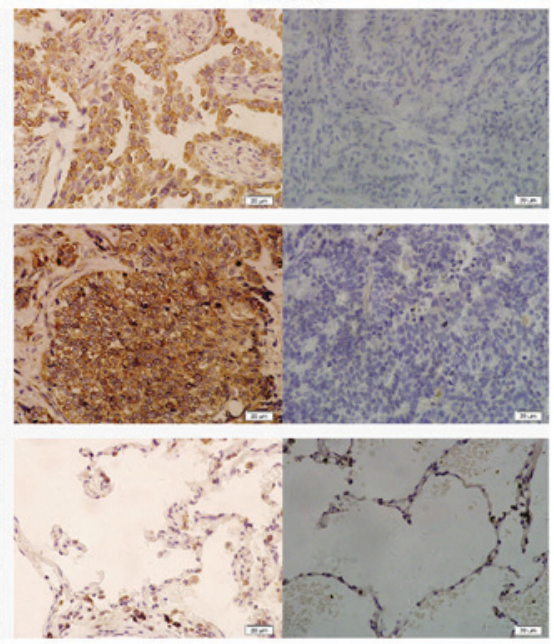

ㅁ NT

Figure 4. (A) Immunohistochemical experiments under an optical microscope (x400): CCL7 and ABCE1 expression was upregulated in lung SCC and AC, as demonstrated by the brown granules in the cytoplasm, and was low in adjacent normal lung tissues. The right side of each group is a negative control. (B) Quantitative immunohistochemical analysis results: The expression of CCL7 and ABCE1 in lung cancer tissues was significantly higher than that in normal lung tissues, "P<0.01. ABCE1, ATP-binding cassette sub-family E member 1; CCL7, chemokine (C-C motif) ligand 7; SCC, squamous cell carcinoma; AC, adenocarcinoma; NT, normal tissue.

$\beta$-actin and that the binding of $\mathrm{ABCE} 1$ to $\beta$-actin requires the Fe-S cluster domain $(14,15)$. These results show that ABCE1 is highly expressed in many malignant tumor cells, indicating that this protein is closely related to the proliferation, invasion and metastasis of lung cancer.

Chemokine CCL7, which was initially identified as a cytokine in mononuclear cells, acts on a variety of target cells, including neutrophils, eosinophils, basophils, natural killer cells, T lymphocytes and other inflammatory cells, as well as dendritic cells and mononuclear cells, particularly mononuclear cells (16). Further research has shown that CCL7 has functions in many diseases. For example, Tsuneyama et al (17) found that CCL7 and mononuclear cell infiltration were present in the portal area of the liver in more than $80 \%$ of patients 

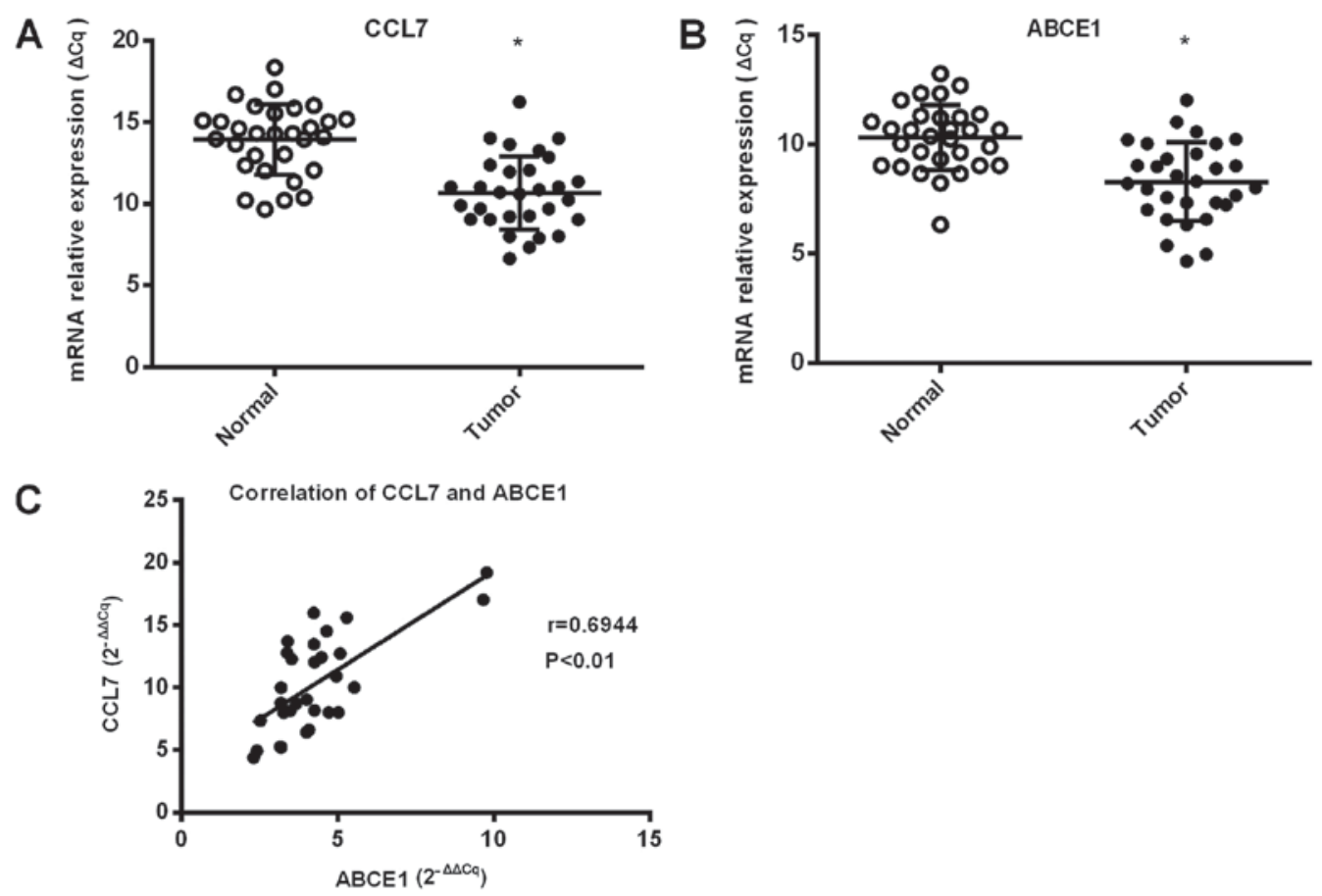

Figure 5. Quantitative PCR experiment. (A) The mRNA expression of CCL7 in lung cancer tissues was significantly higher than that in adjacent NTs, "P<0.01. (B) The mRNA expression of ABCE1 in lung cancer tissues was higher than that in adjacent NTs, "P<0.01. (C) Pearson correlation analysis showed that the expression of CCL7 was positively correlated with that of ABCE1 in lung cancer tissues. ABCE1, ATP-binding cassette sub-family E member 1; CCL7, chemokine (C-C motif) ligand 7; NTs, normal tissues.

with primary biliary cirrhosis, suggesting that elevated CCL7 expression is associated with biliary cirrhosis. The study by Edman et al (18) found that CCL2 and CCL7 selectively enhanced the differentiation of Nurr1+ precursors into dopaminergic (DA) neurons. Gonzalez et al (19) confirmed that CCL7 plays a dual role in renal tubulointerstitial fibrosis by altering the extracellular matrix, an effect that is detrimental at the early stage but beneficial at the later stage.

The role of chemokine CCL7 in tumor growth and metastasis is very complicated, as studies have shown that CCL7 not only promotes tumor metastasis but also inhibits the growth of some malignant tumors $(20,21)$. As CCL7 can play a chemotactic role in many leukocyte subsets, which identify and kill tumor cells, some researchers conducted anti-tumor experiments using mast cells transfected with CCL7. Interestingly, the tumor cells did not die, but the surrounding tumor tissue was infiltrated with TAMs, eosinophils, neutrophils, granulocytes and lymphocytes $(22,23)$. In addition, a large number of dendritic cells accumulated around the peripheral vasculature of the tumor. Wetzel et al found that transfection with a virus containing CCL7 inhibited the growth of cervical cancer cells in humans (24). In contrast, other studies found that CCL7 promoted the brain metastasis of breast cancer cells and was conducive to the growth of cancer cells in the brain, while reduced CCL7 expression inhibited the metastasis of breast cancer cells to the brain (25). Cho et al found that high CCL7 expression is associated with the metastasis of colorectal cancer to the liver (26), and Rajaram et al (27) found that CCL7 plays an important role in promoting the migration and proliferation of tumor cells during the process of mutual transformation involving tumor cells and stromal cells in breast cancer. CCL7 and its receptor, CCR2, promote the brain metastasis of renal cell carcinoma (28). In addition, CCL7 plays an important role in the infiltration and invasion of cancer cells in oral SCC (29).

In the present study, screening using an $\mathrm{RT}^{2}$ Profiler $^{\mathrm{TM}} \mathrm{PCR}$ Array chip showed that the change in the mRNA expression of chemokine CCL7 was significant in NSCLC cell lines that exhibited upregulation of ABCE1, showing that the expression of these two genes is strongly correlated during the processes of NSCLC invasion and metastasis. Western blotting was performed to verify the high expression of CCL7 protein in NSCLC cells that overexpressed ABCE1. The expression of CCL7 and ABCE1 in NSCLC tissues was significantly higher than that in adjacent tissues, as confirmed by immunohistochemistry and qPCR, and a positive correlation between the two genes was observed. These results indicate that CCL7 and ABCE1 are closely associated with the development and metastasis of NSCLC. ABCE1 may change the tumor microenvironment through the chemokine CCL7 pathway; this is a new direction for future research.

\section{Acknowledgements}

Not applicable.

\section{Funding}

This study was supported by the National Natural Science Foundation of China (grant no. 30973502).

\section{Availability of data and materials}

The datasets used and/or analyzed during the current study are available from the corresponding author on reasonable request. 


\section{Authors' contributions}

ZW and DT conceived and designed the study. DT and XY had full access to all the data in the study, and took responsibility for the integrity of the data and the accuracy of the data analysis. ZW, YT, QY, ZT and HL extracted the data. ZW, DT and XY analyzed the data. ZW, DT and XY interpreted the data. ZW, DT and XY wrote the first draft of the manuscript. All authors critically revised the manuscript and approved the final version.

\section{Ethics approval and consent to participate}

Ethical approval was given by the Fourth Affiliated Hospital of China Medical University Ethics Committee. All procedures performed in studies involving human participants were in accordance with the ethical standards of the institutional and/or national research committee and with the 1964 Declaration of Helsinki and its later amendments or comparable ethical standards. All patients provided written informed consent prior to their inclusion in the present study.

\section{Patient consent for publication}

Not applicable.

\section{Competing interests}

The authors declare that they have no competing interests.

\section{References}

1. Bisbal C, Martinand C, Silhol M, Lebleu B and Salehzada T: Cloning and characterization of a RNAse L inhibitor. A new component of the interferon-regulated 2-5A pathway. J Biol Chem 270: 13308-13317, 1995.

2. Lingappa JR, Dooher JE, Newman MA, Kiser PK and Klein KC: Basic residues in the nucleocapsid domain of Gag are required for interaction of HIV-1 gag with ABCE1 (HP68), a cellular protein important for HIV-1 capsid assembly. J Biol Chem 281: 3773-3784, 2006.

3. Huang B, Gao Y, Tian D and Zheng M: A small interfering ABCE1-targeting RNA inhibits the proliferation and invasiveness of small cell lung cancer. Int J Mol Med 25: 687-693, 2010.

4. Tian Y, Tian X, Han X, Chen Y, Song CY, Jiang WJ and Tian DL: ABCE1 plays an essential role in lung cancer progression and metastasis. Tumour Biol 37: 8375-8382, 2016.

5. Zlotnik A and Yoshie O: Chemokines: A new classification system and their role in immunity. Immunity 12: 121-127, 2000.

6. Menten P, Wuyts A and Van Damme J: Monocyte chemotactic protein-3. Eur Cytokine Netw 12: 554-560, 2001.

7. Mishra P, Banerjee D and Ben-Baruch A: Chemokines at the crossroads of tumor-fibroblast interactions that promote malignancy. J Leukoc Biol 89: 31-39, 2011.

8. Torre LA, Bray F, Siegel RL, Ferlay J, Lortet-Tieulent J and Jemal A: Global cancer statistics, 2012. CA Cancer J Clin 65: 87-1083, 2015.

9. Hassel BA, Zhou A, Sotomayor C, Maran A and Silverman RH: A dominant negative mutant of 2-5A-dependent RNase suppresses antiproliferative and antiviral effects of interferon. EMBO J 12 3297-3304, 1993.

10. Chen ZQ, Dong J, Ishimura A, Daar I, Hinnebusch AG and Dean M: The essential vertebrate ABCE1 protein interacts with eukaryotic initiation factors. J Biol Chem 281: 7452-7457, 2006.

11. Barthelme D, Dinkelaker S, Albers SV, Londei P, Ermler U and Tampé R: Ribosome recycling depends on a mechanistic link between the FeS cluster domain and a conformational switch of the twin-ATPase ABCE1. Proc Natl Acad Sci USA 108: 3228-3233, 2011.
12. Ren Y, Li Y and Tian D: Role of the ABCE1 gene in human lung adenocarcinoma. Oncol Rep 27: 965-970, 2012.

13. Gao Y, Xu HH, Wang R, Fang H, Xue YD, Liu JW and Tian DL Expression of a new tumor metastasis-related gene ABCE1 in non-small cell lung cancer and its significance. J Chin Med Univ 40: 911-914, 2011.

14. Han X, Tian Y and Tian D: Tumor metastatic promoter ABCE1 interacts with the cytoskeleton protein actin and increases cell motility. Oncol Rep 35: 3623-3629, 2016.

15. Yu Q, Han X and Tian DL: Deficiency of functional iron-sulfur domains in ABCE1 inhibits the proliferation and migration of lung adenocarcinomas by regulating the biogenesis of beta-actin in vitro. Cell Physiol Biochem 44: 554-566, 2017.

16. Ali S, Robertson H, Wain JH, Isaacs JD, Malik G and Kirby JA: A non-glycosaminoglycan-binding variant of $\mathrm{CC}$ chemokine ligand 7 (monocyte chemoattractant protein-3) antagonizes chemokine-mediated inflammation. J Immunol 175: 1257-1266, 2005.

17. Tsuneyama K, Harada K, Yasoshima M, Hiramatsu K, Mackay CR, Mackay IR, Gershwin ME and Nakanuma Y: Monocyte chemotactic protein-1, -2 , and -3 are distinctively expressed in portal tracts and granulomata in primary biliary cirrhosis: Implications for pathogenesis. J Pathol 193: 102-109, 2001.

18. Edman LC, Mira H and Arenas E: The beta-chemokines CCL2 and CCL7 are two novel differentiation factors for midbrain dopaminergic precursors and neurons. Exp Cell Res 314: 2123-2130, 2008.

19. Gonzalez J, Mouttalib S, Delage C, Calise D, Maoret JJ, Pradère JP, Klein J, Buffin-Meyer B, Van der Veen B, Charo IF, et al: Dual effect of chemokine CCL7/MCP-3 in the development of renal tubulointerstitial fibrosis. Biochem Biophys Res Commun 438: 257-263, 2013.

20. Hwang TL, Lee LY, Wang CC, Liang Y, Huang SF and Wu CM: CCL7 and CCL21 overexpression in gastric cancer is associated with lymph node metastasis and poor prognosis. World J Gastroenterol 18: 1249-1256, 2012.

21. Dempe S, Lavie M, Struyf S, Bhat R, Verbeke H, Paschek S, Berghmans N, Geibig R, Rommelaere J, Van Damme J and Dinsart C: Antitumoral activity of parvovirus-mediated IL-2 and MCP-3/CCL7 delivery into human pancreatic cancer: Implication of leucocyte recruitment. Cancer Immunol Immunother 61: 2113-2123, 2012

22. Fioretti F, Fradelizi D, Stoppacciaro A, Ramponi S, Ruco L, Minty A, Sozzani S, Garlanda C, Vecchi A and Mantovani A: Reduced tumorigenicity and augmented leukocyte infiltration after monocyte chemotactic protein-3 (MCP-3) gene transfer: Perivascular accumulation of dendritic cells in peritumoral tissue and neutrophil recruitment within the tumor. J Immunol 161: 342-346, 1998.

23. Luster AD: Antichemokine immunotherapy for allergic diseases. Curr Opin Allergy Clin Immunol 1: 561-567, 2001.

24. Wetzel K, Menten P, Opdënakker G, Van Damme J, Gröne HJ, Giese N, Vecchi A, Sozzani S, Cornelis JJ, Rommelaere J and Dinsart C: Transduction of human MCP-3 by a parvoviral vector induces leukocyte infiltration and reduces growth of human cervical carcinoma cell xenografts. J Gene Med 3: 326-337, 2001.

25. Wu K, Fukuda K, Xing F, Zhang Y, Sharma S, Liu Y, Chan MD, Zhou X, Qasem SA, Pochampally R, et al: Roles of the cyclooxygenase 2 matrix metalloproteinase 1 pathway in brain metastasis of breast cancer. J Biol Chem 290: 9842-9854, 2015.

26. Cho YB, Lee WY, Choi SJ, Kim J, Hong HK, Kim SH, Choi YL, Kim HC, Yun SH, Chun HK and Lee KU: CC chemokine ligand 7 expression in liver metastasis of colorectal cancer. Oncol Rep 28: 689-694, 2012.

27. Rajaram M, Li J, Egeblad M and Powers RS: System-wide analysis reveals a complex network of tumor-fibroblast interactions involved in tumorigenicity. PLoS Genet 9: e1003789, 2013.

28. Wyler L, Napoli CU, Ingold B, Sulser T, Heikenwälder M, Schraml P and Moch H: Brain metastasis in renal cancer patients: Metastatic pattern, tumour-associated macrophages and chemokine/chemoreceptor expression. Br J Cancer 110: 686-694, 2014.

29. Bae JY, Kim EK, Yang DH, Zhang X, Park YJ, Lee DY, Che CM and Kim J: Reciprocal interaction between carcinoma-associated fibroblasts and squamous carcinoma cells through interleukin-1 $\alpha$ induces cancer progression. Neoplasia 16: 928-938, 2014.

This work is licensed under a Creative Commons Attribution-NonCommercial-NoDerivatives 4.0 International (CC BY-NC-ND 4.0) License. 